\title{
Trajectory and mortality of preserved ratio impaired spirometry: the Rotterdam Study
}

\author{
Sara Renata Alex Wijnant (101,2,3, Emmely De Roos (10 ${ }^{1,2}$, Maryam Kavousi², \\ Bruno Hugo Stricker (10), Natalie Terzikhan ${ }^{2}$, Lies Lahousse $\mathbb{C}^{2,3,6}$ and \\ Guy G. Brusselle (i) ${ }^{1,2,5,6}$
}

Affiliations: ${ }^{1}$ Dept of Respiratory Medicine, Ghent University Hospital, Ghent, Belgium. ${ }^{2}$ Dept of Epidemiology, Erasmus Medical Center, Rotterdam, The Netherlands. ${ }^{3}$ Dept of Bioanalysis, Faculty of Pharmaceutical Sciences, Ghent University, Ghent, Belgium. ${ }^{4}$ Dept of Internal Medicine, Erasmus Medical Center, Rotterdam, The Netherlands. ${ }^{5}$ Dept of Respiratory Medicine, Erasmus Medical Center, Rotterdam, The Netherlands. ${ }^{6}$ Both authors contributed equally.

Correspondence: Guy G. Brusselle, Dept of Respiratory Medicine, University Hospital Ghent, Corneel Heymanslaan 10, 9000 Ghent, Belgium. E-mail: guy.brusselledugent.be

@ERSpublications

Preserved ratio impaired spirometry, previously called restrictive spirometry, is a condition associated with increased mortality that encompasses distinct clinical subsets http://bit.ly/2ncclac

Cite this article as: Wijnant SRA, De Roos E, Kavousi M, et al. Trajectory and mortality of preserved ratio impaired spirometry: the Rotterdam Study. Eur Respir J 2020; 55: 1901217 [https://doi.org/10.1183/ 13993003.01217-2019].

ABSTRACT Preserved ratio impaired spirometry (PRISm) is a heterogeneous condition but its course and disease progression remain to be elucidated. We aimed to examine its prevalence, trajectories and prognosis in the general population.

In the Rotterdam Study (population-based prospective cohort) we examined prevalence, trajectories and prognosis of subjects with normal spirometry (controls; forced expiratory volume in $1 \mathrm{~s}\left(\mathrm{FEV}_{1}\right) /$ forced vital capacity $\left.(\mathrm{FVC}) \geqslant 0.7, \mathrm{FEV}_{1} \geqslant 80 \%\right)$, PRISm $\left(\mathrm{FEV}_{1} / \mathrm{FVC} \geqslant 0.7, \mathrm{FEV}_{1}<80 \%\right)$ and chronic obstructive pulmonary disease $(\mathrm{COPD})\left(\mathrm{FEV}_{1} / \mathrm{FVC}<0.7\right)$ at two study visits. Hazard ratios with $95 \%$ confidence intervals for mortality (until December 30, 2018) were adjusted for age, sex, body mass index, current smoking and pack-years.

Of 5487 subjects (age 69.1 \pm 8.9 years; 7.1\% PRISm), 1603 were re-examined after 4.5 years. Of the re-examined PRISm subjects, $15.7 \%$ transitioned to normal spirometry and $49.4 \%$ to COPD. Median lung function decline was highest in subjects with incident PRISm $\left(\mathrm{FEV}_{1}-92.8 \mathrm{~mL} \cdot \mathrm{year}^{-1}\right.$, interquartile range (IQR) -131.9- -65.8 mL·year ${ }^{-1}$; FVC $-93.3 \mathrm{~mL} \cdot \mathrm{year}^{-1}$, IQR $-159.8--49.1 \mathrm{~mL} \cdot \mathrm{year}^{-1}$ ), but similar in persistent PRISm $\left(\mathrm{FEV}_{1}-30.2 \mathrm{~mL} \cdot \mathrm{year}^{-1}\right.$, IQR -67.9- -7.5 mL·year ${ }^{-1}$; FVC -20.1 mL.year ${ }^{-1}$, IQR -47.7$\left.21.7 \mathrm{~mL} \cdot \mathrm{year}^{-1}\right)$ and persistent controls $\left(\mathrm{FEV}_{1}-39.6 \mathrm{~mL} \cdot \mathrm{year}^{-1}\right.$, IQR $-64.3--12.7 \mathrm{~mL} \cdot \mathrm{year}^{-1}$; FVC -20.0 $\mathrm{mL} \cdot$ year $^{-1}$, IQR $-55.4-18.8 \mathrm{~mL} \cdot \mathrm{year}^{-1}$ ). Of 5459 subjects with informed consent for follow-up, $692(12.7 \%)$ died during 9.3 years (maximum) follow-up: 10.3\% of controls, $18.7 \%$ of PRISm subjects and $20.8 \%$ of COPD subjects. Relative to controls, subjects with PRISm and COPD Global Initiative for Chronic Obstructive Lung Disease (GOLD) 2-4 had increased all-cause mortality (PRISm: HR 1.6, 95\% CI 1.2-2.0; COPD GOLD 2-4: HR 1.7, 95\% CI 1.4-2.1) and cardiovascular mortality (PRISm: HR 2.8, 95\% CI 1.5-5.1; COPD 2-4: HR 2.1, 95\% CI 1.2-3.6). Mortality within <1 year was highest in PRISm, with patients often having cardiovascular comorbidities (heart failure or coronary heart disease; $70.0 \%$ ).

PRISm is associated with increased mortality and this population encompasses at least three distinct subsets: one that develops COPD during follow-up, a second with high cardiovascular burden and early mortality, and a third with persistent PRISm and normal age-related lung function decline.

This article has supplementary material available from erj.ersjournals.com

Received: 20 June 2019 | Accepted after revision: 20 Sept 2019

Copyright $\odot$ ERS 2020 


\begin{abstract}
Introduction
Proportional decreases in forced expiratory volume in $1 \mathrm{~s}\left(\mathrm{FEV}_{1}\right)$ and forced vital capacity (FVC) result in a normal $\mathrm{FEV}_{1} / \mathrm{FVC}$ ratio despite underlying impairment of pulmonary function. Patients with preserved ratio impaired spirometry (PRISm), previously called restrictive pulmonary function $[1,2]$, Global Initiative for Chronic Obstructive Lung Disease (GOLD)-unclassified [3] or the nonspecific pattern $[4,5]$, do not meet the criteria for chronic obstructive pulmonary disease (COPD) but exhibit increased respiratory symptoms $[1,2,6]$, increased cardiovascular comorbidity $[7-15]$ and increased mortality $[6,7$, 16-19]. This neglected subgroup has often been excluded in research papers, though the existing studies to date estimate the prevalence of PRISm to range between $3.0 \%$ and $20 \%$ in adults $[3,5,7,18-23]$. Few of these studies were conducted in the general population and they rarely performed repeated spirometry measurements. Longitudinal studies of subjects with PRISm are scarce and have mostly been conducted in populations of current or past smokers. Some have suggested PRISm is a fluctuating state, serving as an intermediate phase between normal spirometry and COPD for a subset of individuals $[2,3,5,18,20]$. Others have identified risk factors for PRISm, which include female sex [3, 18], smoking, advanced age, metabolic syndrome [24, 25], a history of tuberculosis [26], systemic inflammation [4, 27-29], exposure to fine dust [30] and exposure to biomass cooking fuels [31]. Still, despite receiving increasing attention in pulmonary research, the course and disease progression of PRISm remain to be elucidated.
\end{abstract}

The term PRISm encompasses the findings of impaired spirometry (i.e. decreased $\mathrm{FEV}_{1}$ and FVC) but preserved $\mathrm{FEV}_{1} / \mathrm{FVC}$ ratio. However, a restrictive spirometry pattern can only suggest the presence of a possible pulmonary restriction; this needs confirmation by additional pulmonary function testing that measures residual volume (RV) and total lung capacity (TLC). Given that body plethysmography or multi-breath dilution techniques are time-consuming, costly and need to be completed in a specialised setting, it is reasonable to conduct spirometry in population-based settings [2].

In this paper, we aimed to examine the 1) prevalence, 2) longitudinal patterns of trajectories and 3) prognosis of PRISm in an older general population, including ever- and never-smokers.

\section{Methods}

Study design and study population

This study was conducted within the Rotterdam Study, a population-based cohort study that started in 1989, comprising almost 15000 participants aged $\geqslant 45$ years and aiming to assess the occurrence of, and risk factors for, chronic diseases in the elderly [32]. Every 3-4 years, participants undergo a home interview and clinical examinations at the research centre (supplementary figure S1). The medical ethics committee of the Erasmus Medical Centre (Rotterdam, the Netherlands), and the review board of the Netherlands Ministry of Health, Welfare and Sports (1068889-159521-PG) approved the study. Participants gave written informed consent. The current study contains a cross-sectional and a longitudinal part (figure 1). First, all participants with interpretable spirometry who visited the research

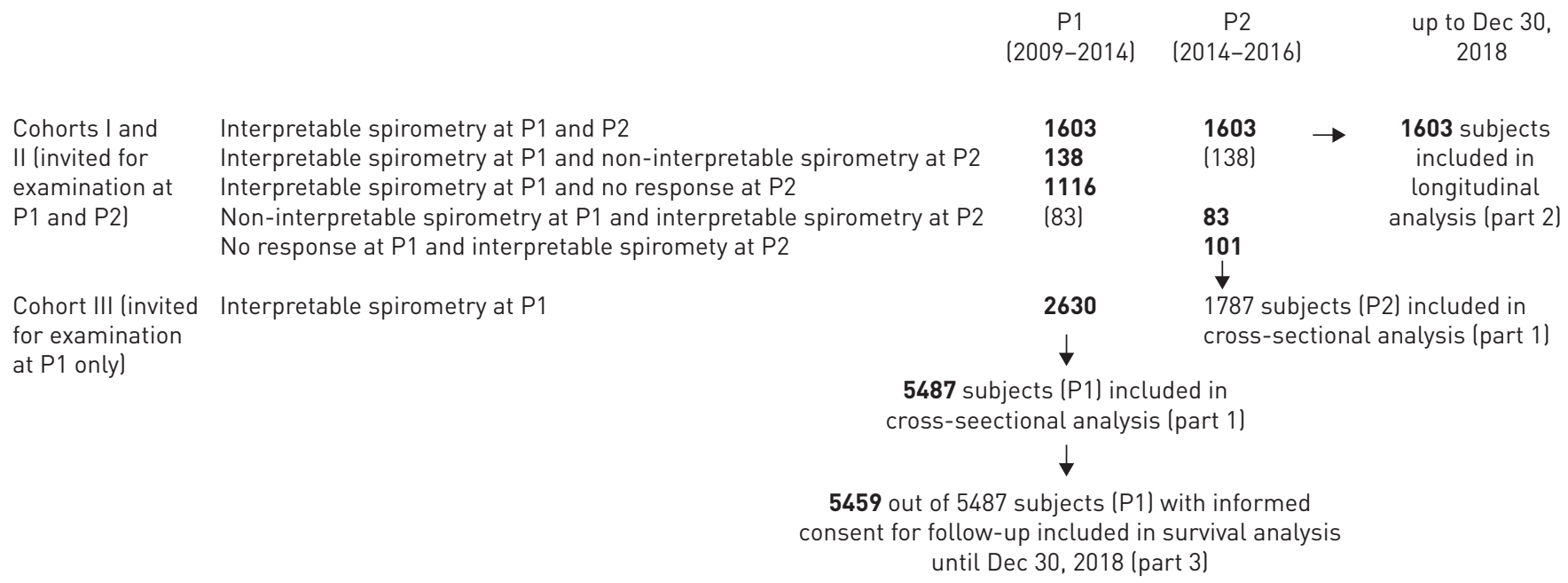

FIGURE 1 Flowchart of participants. Participants with interpretable spirometry who visited the research centre between 2009 and 2014 (phase 1 (P1)) and between 2014 and 2016 (phase 2 (P2)) were eligible for the cross-sectional analysis (part 1). Participants with interpretable spirometry at both P1 and P2 were included in the longitudinal part (part 2). Subjects with interpretable spirometry at P1 and informed consent for follow-up were included in a survival analysis (part 3). 
centre between 2009 and 2014 (phase 1 (P1)) and between 2014 and 2016 (phase 2 (P2)) were eligible for the cross-sectional analysis. Second, participants with interpretable spirometry at both P1 and P2 were included in the longitudinal part. Characteristics of subjects with and without re-examination are presented in supplementary table S1. Third, subjects with interpretable spirometry at P1 and informed consent for follow-up were included in a survival analysis.

\section{Spirometry and diffusion capacity}

Given that body plethysmography or multi-breath dilution techniques were not available in the Rotterdam Study, we used the term PRISm to suggests the presence of a possible pulmonary restriction. Pre-bronchodilator spirometry was performed by trained paramedical personnel using a Master Screen PFT Pro (Care Fusion, Houten, the Netherlands) according to the American Thoracic Society (ATS)/ European Respiratory Society (ERS) guidelines [33]. For diffusing capacity of the lung, the transfer factor using carbon monoxide was used corrected for haemoglobin $\left(D_{\mathrm{LCO}}\right.$ in $\left.\mathrm{mmol} \cdot \mathrm{min}^{-1} \cdot \mathrm{kPA}^{-1}\right)$ [34]. Predicted $\mathrm{FEV}_{1}$ and FVC values were calculated using Global Lung Initiative (GLI) reference equations taking age, sex, height and ethnicity into account [35]. Patients were classified as having PRISm $\left(\mathrm{FEV}_{1} / \mathrm{FVC} \geqslant 70 \%\right.$ and $\left.\mathrm{FEV}_{1}<80 \%\right)$, COPD $\left(\mathrm{FEV}_{1} / \mathrm{FVC}<70 \%\right)$ or normal spirometry (controls; $\mathrm{FEV}_{1} / \mathrm{FVC} \geqslant 70 \%$ and $\mathrm{FEV}_{1} \geqslant 80 \%$ ). The severity of obstruction was determined according to the modified GOLD criteria: mild COPD (GOLD 1, FEV $1 \geqslant 80 \%)$ and moderate to severe COPD (GOLD 2-4, $\mathrm{FEV}_{1}<80 \%$ ).

\section{Characteristics, comorbidities and adverse health outcomes}

Smoking status (never, former and current), cigarette pack-years (years smoked multiplied by daily number of smoked cigarettes divided by 20), body mass index (BMI) (weight in kg divided by height in $\mathrm{m}^{2}$ ), obesity $\left(\mathrm{BMI} \geqslant 30 \mathrm{~kg} \cdot \mathrm{m}^{-2}\right)$, diabetes mellitus (use of blood glucose-lowering medication), hypertension (use of antihypertensive medication, systolic blood pressure $\geqslant 160 \mathrm{mmHg}$ or diastolic blood pressure $\geqslant 100 \mathrm{mmHg}$ ), heart failure (typical symptoms/signs confirmed by radiographic evidence of cardiac dysfunction [36]) and coronary heart disease (myocardial infarction, coronary artery bypass grafting or percutaneous coronary intervention [36]) were compared between cases and controls. Blood samples for determining levels of serum glucose, cholesterol and white blood cell count were obtained at the research centre. Medication prescription data were available through automated pharmacy records. Asthma was based on doctor's diagnosis [37].

Information on vital status was obtained from general practitioners and from municipal records up until December 30, 2018. Causes of death during follow-up in the Rotterdam Study were validated up until January 1, 2015, and were coded according to the International Classification of Diseases (ICD)-10. ICD-10 codes I00-I99 were classified as cardiovascular mortality.

\section{Statistical analysis}

Prevalence was calculated by dividing the total number of cases by the total number of participants. For direct standardisation of age we used the 2013 European Standard Population [38]. Data are expressed as mean $\pm S D$ for normally distributed variables or as median (interquartile range (IQR)) for non-normally distributed variables. Comparisons between subjects with and without PRISm were studied using t-tests (parametrical), Mann-Whitney U tests (non-parametrical) and Chi-squared tests (categorical). Age, sex, BMI, current smoking, log-transformed pack-years, height, total cholesterol, low-density lipoprotein cholesterol, high-density lipoprotein cholesterol, log-transformed triglycerides, haemoglobin, hypertension, heart failure, coronary heart disease and diabetes mellitus were considered as potential confounders in logistic regression analyses. Mortality rates per 1000 person-years were calculated by dividing the number of deaths after spirometry by the total follow-up time of subjects at risk. The mortality rate ratio was calculated by dividing the mortality rate for subgroups by the mortality rate of the reference category. Ageand sex-specific mortality rates were obtained by dividing the number of deaths by the number of person-years accumulated in 10-year age intervals of the study population. We constructed cumulative survival curves using the Kaplan-Meier method. Hazard ratios from Cox proportional hazard models were adjusted for age, sex, BMI, current smoking and pack-years. Proportionality was checked using Schoenfeld residuals. Spirometry results from P2 were included as a time-varying covariate in a sensitivity analysis. We did separate sensitivity analyses excluding asthma subjects and using GLI definitions for $\mathrm{FEV}_{1}$ and FVC with lower limit of normal (LLN) thresholds. Statistical analyses were carried out using R version 3.5.1 (Foundation for Statistical Computing, Vienna, Austria).

\section{Results}

Prevalence of PRISm

Among 14926 participants of the Rotterdam Study, 5487 performed interpretable spirometry tests between 2009 and 2014 (P1; figure 1). 2857 participants were invited for examination between 2014 and 2016, of 
whom 1787 performed interpretable spirometry tests (P2). The crude prevalence of PRISm at P1 (PRISm-P1) was 7.1\% (387 out of 5487). The age-adjusted prevalence of PRISm was $7.6 \%$. Age- and sex-specific prevalence of PRISm and COPD are shown in supplementary figure S2. Table 1 shows characteristics of subjects with PRISm, COPD and normal spirometry at both P1 and P2. A subset of 4759 individuals performed diffusion capacity testing at P1. When adjusted for age, sex, height, log-transformed pack-years, haemoglobin, log-transformed triglycerides, diabetes mellitus and heart failure in linear regression analysis, $D_{\text {LCO }}$ was lower in subjects with PRISm-P1 $(\beta-0.4,95 \%$ confidence interval (CI) $-0.6--0.3, \mathrm{p}<0.001)$ and COPD-P1 $(\beta-0.5,95 \%$ CI-0.6- $-0.4, \mathrm{p}<0.001)$, whereas $D_{\text {LCO divided by }}$ the alveolar volume $\left(V_{\mathrm{A}}\right)$ was higher in subjects with PRISm $(\beta 0.2,95 \%$ CI $0.1-0.2, \mathrm{p}<0.001)$ but lower in subjects with COPD $(\beta-0.1,95 \% \mathrm{CI}-0.1--0.1, \mathrm{p}<0.001)$.

Trajectories of lung function impairment

1603 participants had interpretable spirometry at both phases (P1 and P2; figure 1). Age- and sex-specific cumulative incidence of PRISm and COPD are shown in supplementary figure S2. Subjects with PRISm-P1 showed lower average rates of lung function decline relative to controls and COPD subjects $(\mathrm{p}<0.001$; supplementary table S2). However, subjects with PRISm-P2 showed higher average rates of lung function decline ( $\mathrm{p}<0.001$; table 2 ). Within PRISm-P1 subjects, $10.4 \%$ showed improved lung function (i.e. transitioned to normal spirometry), while $32.6 \%$ transitioned towards COPD (figure 2). PRISm subjects more often transitioned towards a different lung function category (43.0\%) than subjects with normal

TABLE 1 Cross-sectional analysis of characteristics at P1 and P2

\begin{tabular}{|c|c|c|c|c|c|}
\hline \multicolumn{3}{|c|}{ Phase $1^{\#}$} & \multicolumn{3}{|c|}{ Phase $2^{\text {П }}$} \\
\hline $\begin{array}{c}\text { Normal } \\
\text { spirometry-P1 }\end{array}$ & PRISm-P1 & COPD-P1 & $\begin{array}{c}\text { Normal } \\
\text { spirometry-P2 }\end{array}$ & PRISm-P2 & COPD-P2 \\
\hline
\end{tabular}

\begin{tabular}{|c|c|c|c|c|c|c|}
\hline Subjects n & 4185 & 387 & 915 & 1089 & 94 & 604 \\
\hline Age years & $68.8 \pm 8.7$ & $69.8 \pm 9.6^{*}$ & $70.6 \pm 9.1^{*}$ & $78.1 \pm 4.8$ & $79.8 \pm 6.2^{*}$ & $79.2 \pm 5.4^{*}$ \\
\hline Female sex & 2438 (58.3) & 224 (57.9) & $407(44.5)^{*}$ & $666(61.2)$ & 51 (54.3) & $280(46.4)^{*}$ \\
\hline Obesity & $1007(21.4)$ & $156(40.4)^{*}$ & $150(16.4)^{*}$ & $266(26.2)$ & $33(38.4)^{*}$ & $126(21.8)$ \\
\hline BMI kg $\cdot \mathrm{m}^{-2}$ & $27.6 \pm 4.3$ & $29.4 \pm 5.1^{*}$ & $26.5 \pm 4.1^{*}$ & $27.5 \pm 4.2$ & $29.1 \pm 4.6^{*}$ & $26.9 \pm 3.9^{*}$ \\
\hline Height cm & $168.7 \pm 9.4$ & $168.4 \pm 9.8$ & $170.9 \pm 9.8^{*}$ & $165.9 \pm 9.2$ & $166.3 \pm 8.8$ & $168.8 \pm 9.5^{*}$ \\
\hline Caucasian & $4080(97.5)$ & 378 (97.7) & $904(98.8)^{*}$ & $1010(98.4)$ & $85(94.4)^{*}$ & $563(98.8)$ \\
\hline Never-smoker & $1555(37.2)$ & $119(30.7)^{*}$ & $175(19.1)^{*}$ & $417(38.4)$ & $25(26.6)$ & $130(21.5)^{*}$ \\
\hline Former smoker & 2247 (53.7) & $214(55.3)^{*}$ & $504(55.1) *$ & 627 (57.7) & $64(68.1)$ & 408 (67.5)* \\
\hline Current smoker & 383 (9.2) & $54(14.0)^{*}$ & $236(25.8)^{*}$ & $43(4.0)$ & 5 (5.3) & $66(10.9)^{*}$ \\
\hline Pack-years years & $3.0(0.0-18.7)$ & $8.5(0.0-27.3)^{*}$ & $21.0(3.0-41.1) *$ & $1.3(0.0-15.6)$ & $6.0(0.0-25.0)^{*}$ & $12.0(0.1-33.6)^{*}$ \\
\hline Asthma & $334(8.0)$ & $45(11.6)^{*}$ & $107(11.7)^{*}$ & $65(6.0)$ & $16(17.0)^{*}$ & $90(14.9)^{*}$ \\
\hline Total cholesterol mmol- $\mathrm{L}^{-1}$ & $5.5 \pm 1.1$ & $5.3 \pm 1.2^{*}$ & $5.3 \pm 1.1 *$ & NP & NP & NP \\
\hline HDL-cholesterol mmol. $\mathrm{L}^{-1}$ & $1.5 \pm 0.4$ & $1.4 \pm 0.4^{*}$ & $1.5 \pm 0.5$ & NP & NP & NP \\
\hline Triglycerides mmol- $\mathrm{L}^{-1}$ & $1.3(1.0-1.7)$ & $1.4(1.1-1.9)^{*}$ & $1.2(1.0-1.7)$ & NP & NP & NP \\
\hline Glucose $\mathrm{mmol} \cdot \mathrm{L}^{-1}$ & $5.5(5.1-6.0)$ & $5.7(5.2-6.4)^{*}$ & $5.5(5.1-6.0)$ & NP & NP & NP \\
\hline White blood cell count & $6.9 \pm 1.9$ & $7.5 \pm 2.0^{*}$ & $7.6 \pm 2.4^{*}$ & $6.6 \pm 1.9$ & $7.3 \pm 2.7^{*}$ & $6.8 \pm 1.6$ \\
\hline Haemoglobin & $8.8 \pm 0.7$ & $8.7 \pm 0.8^{*}$ & $8.9 \pm 0.8 *$ & $8.7 \pm 0.8$ & $8.7 \pm 0.9$ & $8.8 \pm 0.8 *$ \\
\hline Diabetes mellitus & $338(8.2)$ & $44(11.7)^{*}$ & 90 (10.0) & NP & NP & NP \\
\hline Hypertension & $2992(71.5)$ & $315(81.4)^{*}$ & $686(75.0)^{*}$ & $876(80.4)$ & $80(85.1)$ & $485(80.4)$ \\
\hline Heart failure & 93 (2.2) & $29(7.5)^{*}$ & $57(6.2)^{*}$ & 34 (3.5) & $12(14.5)^{*}$ & $32(5.8)^{*}$ \\
\hline Coronary heart disease & $332(8.0)$ & $49(12.7)^{*}$ & $117(12.8)^{*}$ & 132 (12.2) & 13 (13.8) & $87(14.4)$ \\
\hline FEV $_{1} \%$ pred & $104.0 \pm 13.3$ & $71.7 \pm 7.3^{*}$ & $76.9 \pm 18.4^{*}$ & $105.7 \pm 14.1$ & $71.6 \pm 7.4^{*}$ & $84.3 \pm 18.2^{*}$ \\
\hline FVC \% pred & $101.7 \pm 12.9$ & $71.8 \pm 8.7^{*}$ & $92.3 \pm 17.6^{*}$ & $104.8 \pm 14.5$ & $72.3 \pm 8.6^{*}$ & $100.3 \pm 17.4^{*}$ \\
\hline $\mathrm{FEV}_{1} /$ FVC $\%$ & $78.8 \pm 4.7$ & $76.9 \pm 5.1^{*}$ & $63.2 \pm 6.7^{*}$ & $76.6 \pm 4.6$ & $75.0 \pm 4.1^{*}$ & $63.0 \pm 7.1^{*}$ \\
\hline$D_{\text {Lco }} \mathrm{mmol} \cdot \mathrm{min}^{-1} \cdot \mathrm{kPA}^{-1}$ & $7.9 \pm 2.0$ & $7.5 \pm 1.6^{*}$ & $7.4 \pm 2.0^{*}$ & NP & NP & NP \\
\hline$D_{\text {Lco }} \%$ pred & $97.0 \pm 14.0$ & $91.5 \pm 17.6^{*}$ & $88.6 \pm 18.4^{*}$ & NP & NP & NP \\
\hline$D_{\mathrm{Lco}} / V_{\mathrm{A}} \mathrm{mmol} \cdot \mathrm{min}^{-1} \cdot \mathrm{kPA}^{-1} \cdot \mathrm{mL}^{-1}$ & $1.5 \pm 0.2$ & $1.7 \pm 0.2^{*}$ & $1.4 \pm 0.3^{*}$ & NP & NP & NP \\
\hline$D_{\text {Lco }} / V_{\mathrm{A}} \%$ pred & $110.0 \pm 16.3$ & $121.5 \pm 20.5^{*}$ & $100.7 \pm 20.2^{*}$ & NP & NP & NP \\
\hline
\end{tabular}

Data are presented as mean \pm SD, $n(\%)$ or median (interquartile range). P1: phase 1; P2: phase 2; PRISm: preserved ratio impaired spirometry; COPD: chronic obstructive pulmonary disease; BMI: body mass index; HDL: high-density lipoprotein; FEV ${ }_{1}$ : forced expiratory volume in 1 s; FVC: forced vital capacity; $D_{\mathrm{LCO}}$ : diffusing capacity of the lung for carbon monoxide; $V_{\mathrm{A}}$ : alveolar volume; NP: not performed. \#: $\mathrm{n}=5487$; ๆ: $\mathrm{n}=1787 ;{ }^{*}$ : $\mathrm{p}<0.05$ compared to subjects with normal spirometry. 
TABLE 2 Lung function values at P1 and P2 stratified by lung function category and smoking status at P2

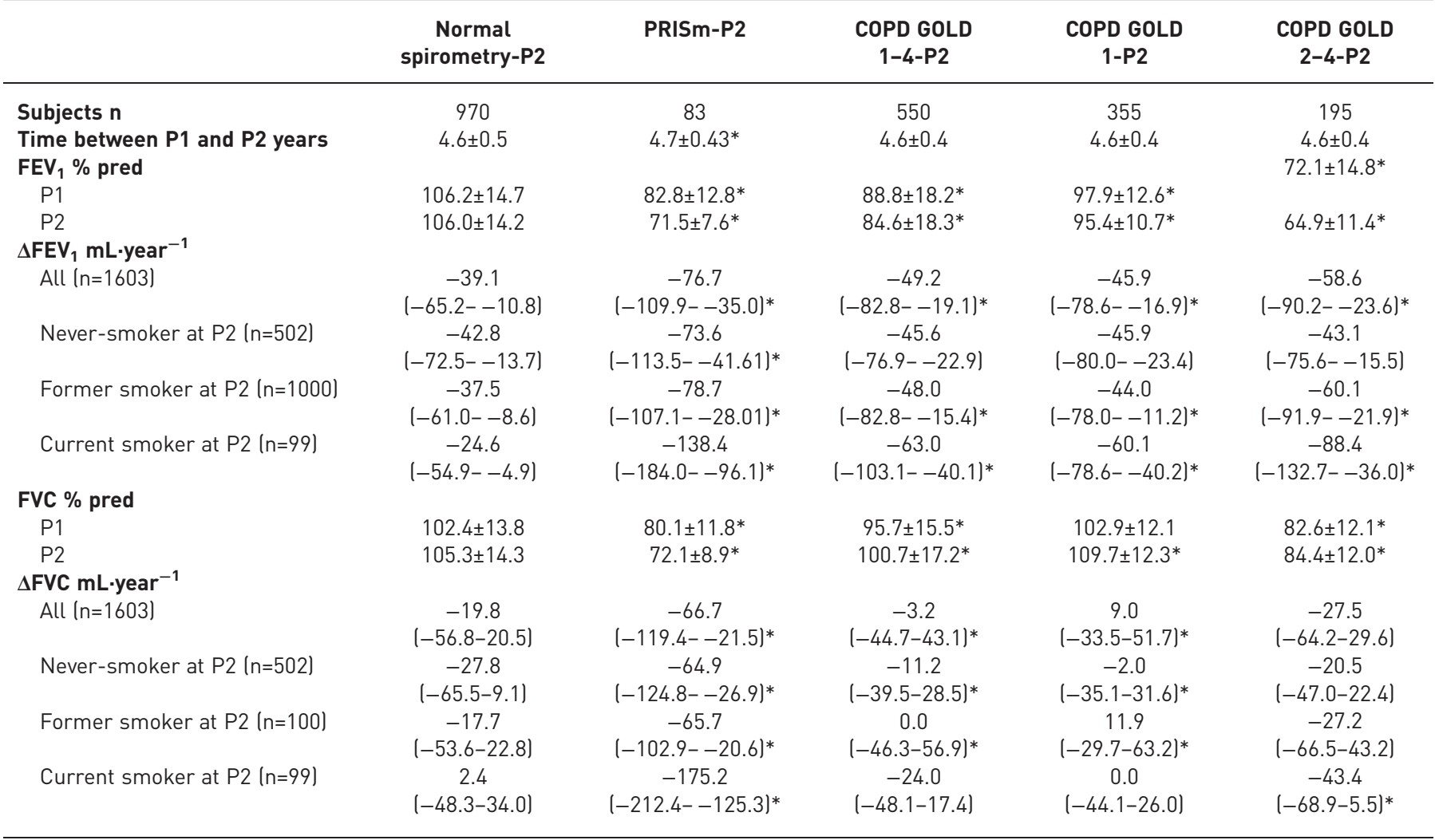

Data are presented as mean \pm SD or median (interquartile range). P1: phase 1; P2: phase 2; PRISm: preserved ratio impaired spirometry; COPD: chronic obstructive pulmonary disease; GOLD: Global Initiative for Chronic Obstructive Lung Disease; FEV ${ }_{1}$ : forced expiratory volume in $1 \mathrm{~s}$; FVC: forced vital capacity. *: p<0.05 compared to subjects with normal spirometry.

a)

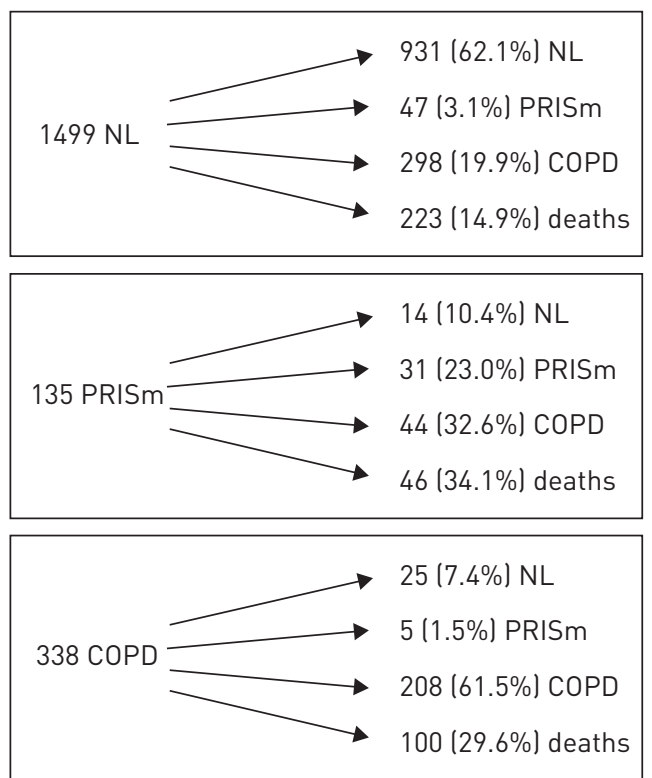

b)

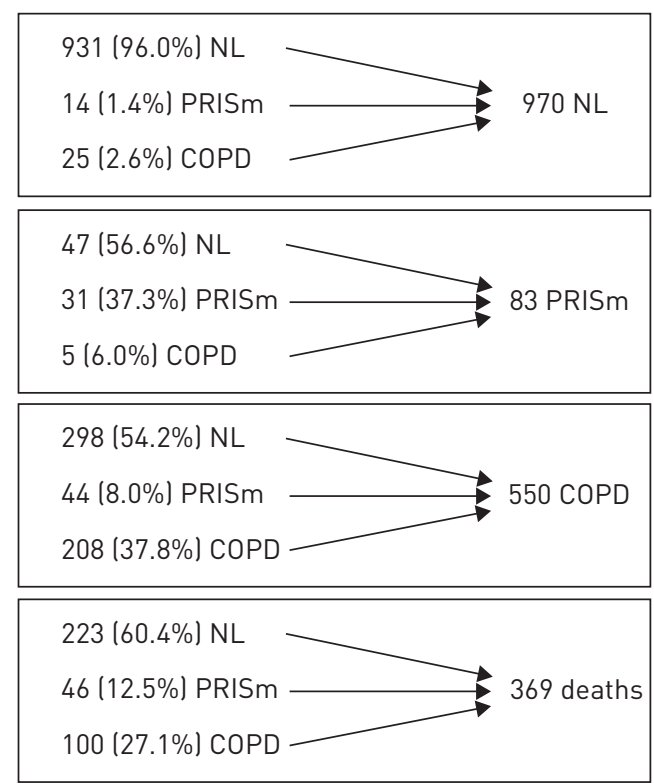

FIGURE 2 Transitions of lung function categories between al phase 1 and b) phase 2. NL: normal spirometry; PRISm: preserved ratio impaired spirometry; COPD: chronic obstructive pulmonary disease. 
(23.0\%) or obstructive lung function (8.9\%). Current smoking accelerated the $\mathrm{FEV}_{1}$ and FVC decline in subjects with PRISm and COPD (table 2; supplementary table S2).

\section{Characteristics of subjects with PRISm by different trajectories}

Subjects with persistent PRISm (i.e. PRISm-P1 and PRISm-P2) had the highest mean BMI at baseline $\left(30.2 \pm 4.1 \mathrm{~kg} \cdot \mathrm{m}^{-2}, \mathrm{p}<0.001\right.$; supplementary table S3). Baseline FVC \% pred, but not $\mathrm{FEV}_{1} \%$ pred, was lower in persistent PRISm than in subjects who evolved from PRISm-P1 towards COPD at P2 ( $\mathrm{p}=001$; supplementary table S3). PRISm-P1 subjects with normal spirometry at P2 were more often female $(\mathrm{p}=0.041)$, had a smaller waist circumference at $\mathrm{P} 1(\mathrm{p}=0.031)$ and had smoked fewer pack-years $(\mathrm{p}=0.098)$ than other subjects with PRISm-P1 (supplementary table S3). In a subgroup analysis of 1390 individuals with diffusion capacity testing performed at $\mathrm{P} 1, D_{\mathrm{LCO}}$ and $D_{\mathrm{LCO}} / V_{\mathrm{A}}$ values did not differ significantly between subjects with persistent PRISm versus incident COPD (i.e. PRISm-P1 and COPD-P2) or incident normal spirometry (i.e. PRISm-P1 and normal spirometry at P2; supplementary table S4), even after adjustment for potential confounders.

\section{Persistent PRISm versus persistent controls and persistent COPD}

Median $\mathrm{FEV}_{1}$ and FVC decline rates were similar in subjects with persistent PRISm $\left(\mathrm{FEV}_{1}:-31.0 \mathrm{~mL} \cdot \mathrm{year}^{-1}\right.$, IQR -69.6- -7.7 mL·year ${ }^{-1}$; FVC: $-20.6 \mathrm{~mL} \cdot$ year $^{-1}$, IQR $-48.9-22.2 \mathrm{~mL} \cdot$ year $^{-1}$ ) and subjects with persistent normal spirometry $\left(\mathrm{FEV}_{1}\right.$ : $-40.6 \mathrm{~mL} \cdot \mathrm{year}^{-1}$, IQR $-65.9--13.0 \mathrm{~mL} \cdot \mathrm{year}^{-1}$; FVC: $-20.5 \mathrm{~mL} \cdot \mathrm{year}^{-1}$, IQR -56.8-19.3 mL·year ${ }^{-1}$ ) (supplementary table S4). Decline rates for FVC, but not $\mathrm{FEV}_{1}$, were lower in subjects with persistent COPD $\left(\mathrm{FEV}_{1}\right.$ : $-36.2 \mathrm{~mL} \cdot \mathrm{year}^{-1}$, IQR $-64.0--4.1 \mathrm{~mL} \cdot \mathrm{year}^{-1}$; FVC: $7.1 \mathrm{~mL} \cdot \mathrm{year}^{-1}$, IQR $\left.-39.8-56.3 \mathrm{~mL} \cdot \mathrm{year}^{-1}\right)$ than in subjects with persistent PRISm $(\mathrm{p}<0.001)$. Subjects with persistent PRISm had more features of metabolic syndrome (higher BMI, waist circumference and glucose; $\mathrm{p}<0.05$ ) and more cardiovascular comorbidity (incident heart failure; $\mathrm{p}<0.05$ ) than subjects with persistent normal spirometry or persistent COPD (supplementary table S3). $D_{\mathrm{LCO}}$ did not differ significantly between these subgroups. $D_{\mathrm{LCO}} /$ $V_{\mathrm{A}}$ was significantly higher in subjects with persistent PRISm than in subjects with persistent normal spirometry $(\mathrm{p}<0.001)$ or persistent COPD $(\mathrm{p}<0.001$; supplementary table $\mathrm{S} 4)$.

\section{Incident PRISm versus incident COPD}

Subjects who developed incident PRISm (i.e. normal spirometry or COPD at P1 and PRISm at P2) were older ( $p=0.001)$, had higher BMI $(p=0.010)$, higher waist circumference $(p=0.005)$, worse pulmonary function $(\mathrm{p}<0.001)$, higher $D_{\mathrm{LCO}} / V_{\mathrm{A}}$ values $(\mathrm{p}<0.001)$, more often gained weight $\left(\Delta \mathrm{BMI}>1.5 \mathrm{~kg} \cdot \mathrm{m}^{-2} ; \mathrm{p}=0.007\right)$ and more often developed incident heart failure $(\mathrm{p}<0.001)$ than other subjects (table 3$)$. Overall, higher $D_{\mathrm{LCO}} / V_{\mathrm{A}}$ values were seen in both prevalent and incident PRISm subjects compared to other lung function groups (supplementary table S4). After adjustment for age, sex, baseline BMI, current smoking and pack-years, a $1.5 \mathrm{~kg} \cdot \mathrm{m}^{-2}$ increase in BMI (OR 3.0, 95\% CI 1.5-5.7, $\mathrm{p}<0.001$ ) and incident heart failure (OR 8.0, 95\% CI 2.2-25.4, $\mathrm{p}<0.001)$ were independently associated with incident PRISm. Subjects with incident PRISm also had the largest decline in $\mathrm{FEV}_{1}$ and FVC $\left(\mathrm{FEV}_{1}\right.$ : $-95.2 \mathrm{~mL} \cdot \mathrm{year}^{-1}, \mathrm{IQR}-135.2--67.5 \mathrm{~mL} \cdot \mathrm{year}^{-1}$; FVC: $-95.7 \mathrm{~mL} \cdot$ year $^{-1}$, IQR $-163.9--50.4 \mathrm{~mL} \cdot$ year $\left.^{-1}\right)$, even relative to incident COPD $\left(\mathrm{FEV}_{1}:-58.5 \mathrm{~mL} \cdot \mathrm{year}^{-1}\right.$, IQR -91.3- -28.6 mL-year ${ }^{-1}$; FVC: $-11.5 \mathrm{~mL} \cdot$ year $^{-1}$, IQR $-47.7-35.4 \mathrm{~mL} \cdot \mathrm{year}^{-1}$; $\left.\mathrm{p}<0.001\right)$. Subjects with incident COPD were older at baseline $(p<0.001)$, were more often male $(p<0.001)$ and current smokers $(p<0.001)$ and more often developed incident coronary heart disease $(p=0.034)$ than other subjects (supplementary table S3). Compared to subjects with persistent normal spirometry, the mortality rate ratio was highest in subjects with incident PRISm (3.8), followed by persistent PRISm (3.2), persistent COPD (1.8) and incident COPD (1.4; supplementary table S5).

Subjects with incident PRISm and normal spirometry at P1 (90\% of incident PRISm; figure 2) differed from subjects with incident COPD and normal spirometry at P1 by their lower $\mathrm{FEV}_{1} \%$ pred and markedly lower FVC \% pred at P1 ( $<<0.001$; supplementary table S3). They had a higher BMI (incident PRISm: $29.0 \pm 4.8 \mathrm{~kg} \cdot \mathrm{m}^{-2}$; incident COPD: $27.2 \pm 3.7 \mathrm{~kg} \cdot \mathrm{m}^{-2}$; $\mathrm{p}=0.003$ ), a higher waist circumference (incident PRISm: $97.2 \pm 14.5 \mathrm{~cm}$; incident COPD: $92.9 \pm 11.7 \mathrm{~cm} ; \mathrm{p}=0.025$ ), more often gained weight $\left(\triangle \mathrm{BMI}>1.5 \mathrm{~kg} \cdot \mathrm{m}^{-2}\right)$ between P1 and P2 (incident PRISm: 25.5\%; incident COPD: 10.7\%; $\mathrm{p}=0.010$ ), had lower high-density lipoprotein cholesterol (incident PRISm: $1.4 \pm 0.3$; incident COPD: $1.5 \pm 0.4 \mathrm{mmol} \cdot \mathrm{L}^{-1}$; $\mathrm{p}=0.046$ ) and more often developed comorbid heart failure between P1 and P2 (incident PRISm: 10.9\%; incident COPD: 0.7\%; $<<0.001$; supplementary table S4).

\section{Prognosis of PRISm}

Within 9.8 years (maximum) of follow-up starting from P1, 692 out of 5459 subjects (12.7\%) with informed consent for follow-up died (figure 1): 430 subjects with normal spirometry (10.3\%), 72 subjects with PRISm (18.7\%) and 190 subjects with COPD (20.8\%). Subjects with PRISm had unadjusted mortality rates similar to those with COPD (figure 3a), which were intermediate between COPD GOLD 1 and GOLD 2-4 (figure 3b). 
TABLE 3 Baseline characteristics at P1 of subjects with incident PRISm compared to characteristics of other subjects (prevalent PRISm and COPD excluded)

Other subjects-P1 (prevalent PRISm and

Incident PRISm-P2 COPD excluded)

\begin{tabular}{|c|c|c|}
\hline Subjects $\mathrm{n}$ & 1229 & 52 \\
\hline Time between $P 1$ and $P 2$ years & $5.5 \pm 1.1$ & $6.1 \pm 1.4^{*}$ \\
\hline Age years & $73.6 \pm 4.8$ & $75.9 \pm 5.9 *$ \\
\hline Female sex & $702(57.1)$ & $29(55.8)$ \\
\hline \multicolumn{3}{|l|}{$\mathrm{BMI} \mathrm{kg} \cdot \mathrm{m}^{-2}$} \\
\hline P1 & $27.5 \pm 4.1$ & $29.0 \pm 4.7^{*}$ \\
\hline P2 & $27.4 \pm 4.1$ & $29.1 \pm 5.0$ \\
\hline$\Delta \mathrm{BMI} \mathrm{kg} \cdot \mathrm{m}^{-2}$ & $-0.14 \pm 1.54$ & $0.05 \pm 2.49$ \\
\hline$\Delta \mathrm{BMI}>1.5 \mathrm{~kg} \cdot \mathrm{m}^{-2} \%$ & $141(11.5)$ & $13(25.0)^{*}$ \\
\hline \multicolumn{3}{|l|}{ Waist circumference $\mathrm{cm}$} \\
\hline P1 & $92.9 \pm 11.7$ & $97.6 \pm 14.7^{*}$ \\
\hline $\mathrm{P} 2$ & $94.7 \pm 12.2$ & $101.2 \pm 15.2^{*}$ \\
\hline$\Delta$ waist circumference $\mathrm{cm}$ & $1.8 \pm 6.6$ & $3.5 \pm 7.2$ \\
\hline$\Delta$ waist circumference $>5 \mathrm{~cm} \%$ & $309(25.1)$ & $21(40.4)^{*}$ \\
\hline Never-smoker & 453 (36.9) & $16(30.8)$ \\
\hline Former smoker & $695(56.6)$ & 32 (61.5) \\
\hline Current smoker & $81(6.6)$ & $4(7.7)$ \\
\hline Pack-years & $3.2(0.0-18.8)$ & $3.0(0.0-21.8)$ \\
\hline Total cholesterol mmol. $\mathrm{L}^{-1}$ & $5.5 \pm 1.1$ & $5.4 \pm 1.2$ \\
\hline HDL-cholesterol $\mathrm{mmol} \cdot \mathrm{L}^{-1}$ & $1.5 \pm 0.4$ & $1.4 \pm 0.3^{*}$ \\
\hline Triglycerides $\mathrm{mmol} \cdot \mathrm{L}^{-1}$ & $1.3(1.0-1.7)$ & $1.3(1.1-1.6)$ \\
\hline Glucose mmol. $\mathrm{L}^{-1}$ & $5.5(5.1-6.1)$ & $5.7(5.2-6.5)^{*}$ \\
\hline White blood cell count & $6.8 \pm 1.6$ & $7.0 \pm 2.0$ \\
\hline Diabetes mellitus & $91(7.5)$ & $6(11.5)$ \\
\hline Hypertension & $1025(83.4)$ & 45 (86.5) \\
\hline Prevalent heart failure & $33(2.7)$ & $1(1.9)$ \\
\hline Incident heart failure & $10(0.8)$ & $5(9.8)^{*}$ \\
\hline Prevalent coronary heart disease & $118(9.6)$ & $5(9.6)$ \\
\hline Incident coronary heart disease & $25(2.3)$ & $1(2.1)$ \\
\hline $\mathrm{FEV}_{1} \%$ pred & $105.3 \pm 13.7$ & $89.3 \pm 11.1^{*}$ \\
\hline$\Delta \mathrm{FEV}_{1} \mathrm{~mL} \cdot \mathrm{year}^{-1}$ & $-44.7(-74.9--16.8)$ & $-95.2(-135.2--67.5)^{*}$ \\
\hline FVC $\%$ pred $\%$ & $102.5 \pm 13.0$ & $86.7 \pm 8.5^{*}$ \\
\hline$\Delta \mathrm{FVC} \mathrm{mL-year^{-1 }}$ & $-18.2(-54.7-22.6)$ & $-95.7(-163.0--50.4)^{*}$ \\
\hline $\mathrm{FEV}_{1} / \mathrm{FVC} \%$ & $78.6 \pm 4.7$ & $78.2 \pm 5.4$ \\
\hline$\Delta \mathrm{FEV}_{1} / \mathrm{FVC} \%$ & $-1.0 \pm 1.1$ & $-0.7 \pm 1.3$ \\
\hline$D_{\text {Lco }} \mathrm{mmol} \cdot \mathrm{min}^{-1} \cdot \mathrm{kPA}^{-1}$ & $7.5 \pm 1.5$ & $7.3 \pm 1.3$ \\
\hline$D_{\text {Lco }} \%$ pred & $97.3 \pm 14.0$ & $96.6 \pm 17.3$ \\
\hline$D_{\mathrm{Lco}} / V_{\mathrm{A}} \mathrm{mmol} \cdot \mathrm{min}^{-1} \cdot \mathrm{kPA}^{-1} \cdot \mathrm{mL}^{-1}$ & $1.5 \pm 0.2$ & $1.6 \pm 0.2^{*}$ \\
\hline$D_{\mathrm{Lco}} / V_{\mathrm{A}} \%$ pred & $111.5 \pm 16.4$ & $121.5 \pm 17.1^{*}$ \\
\hline
\end{tabular}

Data are presented as mean \pm SD, $n(\%)$ and median (interquartile range). P1: phase 1; PRISm: preserved ratio impaired spirometry; COPD: chronic obstructive pulmonary disease; P2: phase 2; BMI: body mass index; $\mathrm{FEV}_{1}$ : forced expiratory volume in $1 \mathrm{~s}$; FVC: forced vital capacity; $D_{\mathrm{LCO}}$ : diffusing capacity of the lung for carbon monoxide; $V_{A}$ : alveolar volume. ${ }^{*}: p<0.05$ compared to subjects without incident PRISm.

Mortality within 1 year after examination was highest in subjects with PRISm $(n=10)$. They more often had cardiovascular comorbidity (heart failure and/or coronary heart disease; 70.0\%) compared to subjects with PRISm who died later ( $\geqslant 1$ year; $27.4 \%$ ). Of the subjects that died within 1 year after the examination, cardiovascular comorbidity was lower in subjects with normal spirometry (20.0\%) or COPD (44.4\%) than in subjects with PRISm. Age- and sex-specific mortality are shown in supplementary figure S2.

Relative to controls, PRISm and COPD GOLD 2-4 were significant predictors of all-cause mortality in adjusted Cox proportional hazard survival analyses (table 4). Additional adjustment for cardiovascular comorbidity did not affect these associations (supplementary table S5). Disease-specific mortality was known for 285 out of 692 deaths (cardiovascular mortality: 40.6\% in PRISm, 30.8\% in COPD, 25.3\% in controls; supplementary table S6). PRISm and COPD GOLD 2-4 were significant predictors of cardiovascular mortality (table 4). Within subjects with PRISm, stratification by FVC severity, but not by $\mathrm{FEV}_{1}$ severity, distinguished subsets with and without increased cardiovascular mortality (table 4). 

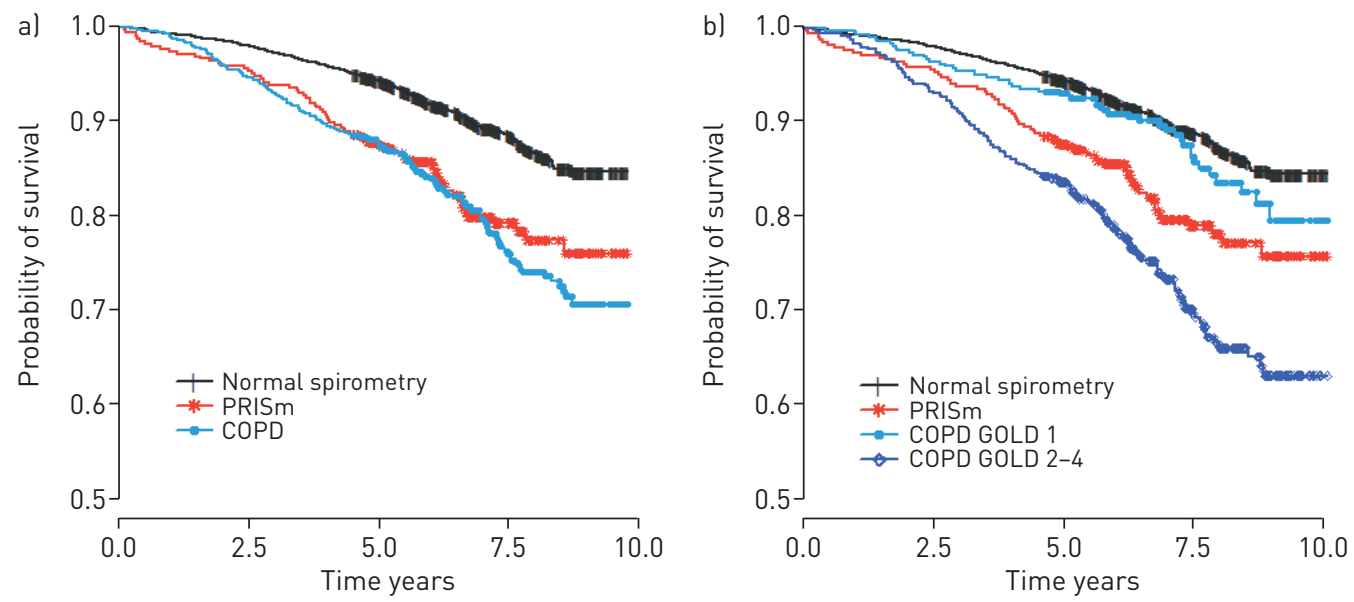

FIGURE 3 Kaplan-Meier curve of mortality for different lung function groups. PRISm: preserved ratio impaired spirometry; COPD: chronic obstructive pulmonary disease; GOLD: Global Initiative for Chronic Obstructive Lung Disease.

\section{Sensitivity analysis}

When using LLN thresholds for both $\mathrm{FEV}_{1}$ and $\mathrm{FEV}_{1} / \mathrm{FVC}$, the prevalence of PRISm-P1 was lower (5.1\% versus $7.1 \%$ ). $19.6 \%$ versus $10.4 \%$ of PRISm-P1 subjects transitioned towards normal spirometry, while $20.6 \%$ versus $32.6 \%$ transitioned towards COPD (supplementary figure S3). When using LLN definitions in adjusted survival analysis (versus GOLD definitions), relative to controls PRISm (HR 1.9, 95\% CI 1.52.5 versus HR 1.6, 95\% CI 1.2-2.0) and COPD GOLD 2-4 (HR 1.9, 95\% CI 1.5-2.5 versus HR 1.7, 95\% CI 1.4-2.1) were significant predictors of all-cause mortality (supplementary table S5). When excluding asthma subjects $(n=486)$, the prevalence, progression and prognosis of subjects with PRISm remained unchanged (supplementary table S5). When including spirometry results from P2 as a time-dependent covariate in the Cox proportional hazard survival analysis, all-cause and cardiovascular mortality in subjects with PRISm remained unchanged (table 4).

\section{Discussion}

PRISm was found in $7.1 \%$ of older participants of the Rotterdam Study and showed higher all-cause and cardiovascular mortality, as well as frequent transition towards COPD. Repeated spirometry measurements were available for this study. Previous longitudinal studies on PRISm mainly included cohorts that had single spirometry measurements at baseline with outcomes of mortality [6, 7, 16-19] or incident comorbid

TABLE 4 Estimates of mortality stratified by lung function category at P1

\begin{tabular}{|c|c|c|c|c|c|c|}
\hline & & $\begin{array}{l}\text { Number } \\
\text { at risk }\end{array}$ & $\operatorname{HR}(95 \% \mathrm{CI})^{\#}$ & $\operatorname{HR}(95 \% \mathrm{CI})^{\#, \text { I }}$ & $\operatorname{HR}(95 \% \mathrm{Cl})^{+}$ & $\operatorname{HR}(95 \% \mathrm{CI})^{\uparrow,+}$ \\
\hline \multicolumn{2}{|c|}{ Normal spirometry-P1 } & 4150 & Reference & Reference & Reference & Reference \\
\hline \multicolumn{2}{|c|}{ PRISm-P1 } & 384 & $1.6(1.2-2.0)^{*}$ & $1.6(1.2-2.0)^{*}$ & $2.7(1.5-5.0)^{*}$ & $2.6(1.4-4.7) *$ \\
\hline \multirow{2}{*}{$\begin{array}{l}\text { PRISm by FEV } \\
\text { severity }\end{array}$} & $F E V_{1} \geqslant 74 \%$ & 194 & $1.3(0.9-1.8)$ & $1.4(1.0-1.9)$ & $2.6(1.1-5.7)^{*}$ & $2.5(1.1-5.5)^{*}$ \\
\hline & $F E V_{1}<74 \%$ & 190 & $1.9(1.4-2.6)^{*}$ & $1.7(1.3-2.3)^{*}$ & $2.8(1.3-6.0)^{*}$ & $2.6(1.2-5.7)^{*}$ \\
\hline \multirow{2}{*}{$\begin{array}{c}\text { PRISm by FVC } \\
\text { severity }\end{array}$} & $F V C \geqslant 73 \%$ & 191 & $1.4(1.0-2.1)$ & $1.4(0.9-2.0)$ & $1.8(0.7-5.1)$ & $1.7(0.6-4.8)$ \\
\hline & $F V C<73 \%$ & 193 & $1.7(1.2-2.3)^{*}$ & $1.7(1.3-2.3)^{*}$ & $3.2(1.6-6.4)^{*}$ & $3.1(1.6-6.1)^{*}$ \\
\hline \multicolumn{2}{|c|}{ COPD GOLD 1-P1 } & 419 & $1.0(0.7-1.3)$ & $0.7(0.5-0.9)^{*}$ & $0.5(0.2-1.6)$ & $0.5(0.1-1.5)$ \\
\hline \multicolumn{2}{|c|}{ COPD GOLD 2-4-P1 } & 493 & $1.7(1.4-2.1)^{*}$ & $1.5(1.2-1.8)^{*}$ & $1.9(1.1-3.4)^{*}$ & $1.8(1.0-3.1)^{*}$ \\
\hline
\end{tabular}

Italic text shows PRISm values stratified by median values for $\mathrm{FEV}_{1}$ and $\mathrm{FVC}$ within PRISm, respectively. P1: phase 1; HR: hazard ratio; PRISm: preserved ratio impaired spirometry; $F E V_{1}$ : forced expiratory volume in $1 \mathrm{~s}$; FVC: forced vital capacity; COPD: chronic obstructive pulmonary disease; GOLD: Global Initiative for Chronic Obstructive Lung Disease. \#: HR for all-cause mortality adjusted for age, sex, body mass index, current smoking and pack-years; ": time-dependent Cox proportional hazard model with lung function category at phase 2 as time varying covariate (subjects with missing information for one of the covariates were excluded $\left(n=13\right.$, of whom six died)); ${ }^{+}$: HR for cardiovascular mortality adjusted for age, sex, body mass index, current smoking and pack-years; *: p<0.05 compared to subjects without incident PRISm. 
disease [8-14, 21, 22]. Longitudinal cohort studies with repeated spirometry measurements investigating trajectories are scarce and were mostly conducted in smoking populations $[1-3,5,18]$. This study provides added value in elucidating the course and disease progression of PRISm in a population-based prospective cohort study encompassing not only smokers (former and current) but also never-smokers.

In this study, a third of subjects with PRISm at baseline transitioned towards COPD at follow-up, while half of subjects with PRISm at follow-up had normal spirometry at baseline. A transition from PRISm to COPD was observed in 15\%-38\% of subjects from the COPDgene study, the Lovelace Smoker's Cohort, the Mayo clinic and the TESOAD study $[2,3,5,18]$. Most studies in the USA and Europe have shown prevalences of PRISm ranging between $3 \%$ and $20 \%$ [3, 5, 7, 18-21, 23]. Using poorly executed spirometry as an explanation for spirometry results suggestive of a restrictive syndrome cannot account for such high prevalence. These findings support the hypothesis that PRISm represents a distinct entity, and is a transitional stage between normal spirometry and COPD in a subset of individuals.

Results from our study were similar to those of the COPDgene study; subjects with incident PRISm showed the largest declines in FVC, while subjects who transitioned from PRISm to COPD showed a smaller decline in FVC but a more severe decline in $\mathrm{FEV}_{1}$ [18]. We more often observed a direct transition from normal spirometry to COPD than to PRISm. It is possible that both situations represent distinct disease courses, whereby FVC decline in PRISm depends on factors other than those in COPD. Literature on the association between smoking and PRISm is inconclusive; some studies have shown a link with PRISm $[3,8,19,20]$, while others have not $[2,5,16]$. We found that smoking affected $\mathrm{FEV}_{1}$ and FVC decline in subjects with PRISm and COPD. Lung function trajectories were only determined in subjects who survived until P2; therefore, the actual decline rates are likely underestimated. Other than age and smoking, possible risk factors and markers of FVC decline in subjects with PRISm are the presence of respiratory symptoms [1], a positive bronchodilator response [5], impaired slow vital capacity [5], physical inactivity [27], metabolic syndrome [27] and systemic inflammation [4, 27, 28]. Additional longitudinal studies with frequent repeated spirometry testing and long-term follow-up are desirable to capture all phases of pulmonary function decline.

Studies have consistently shown increased mortality in subjects with PRISm [6, 7, 16-19]. Our study confirms previous findings and shows mortality risks in PRISm similar to those in COPD GOLD 2-4. We add to existing knowledge by investigating mortality in subjects with PRISm in the general population, encompassing smokers and non-smokers, and by examining cardiovascular mortality, which was higher in PRISm (HR 2.7) than in COPD (HR 1.9). Previous studies have found similar effect estimates but differed in their definition of PRISm [2, 19]. Moreover, impairment of FVC in subjects with PRISm appeared to better distinguish a subset with higher cardiovascular mortality than impairment in $\mathrm{FEV}_{1}$. Interestingly, subjects with PRISm who died within 1 year after spirometry often had comorbid heart failure and/or coronary heart disease. Pulmonary congestion and cardiomegaly are mechanisms by which heart failure could result in pulmonary restriction [39, 40]. Moreover, reduced lung volumes and atelectasis are common after open-heart surgery for coronary heart disease [41].

The high prevalence of heart failure and obesity in subjects with PRISm in this study and previous studies $[7,14,16,25,42-44]$ likely contributes to the low $D_{\mathrm{LCO}}$ values and supranormal $D_{\mathrm{LCO}} / V_{\mathrm{A}}$ values we observed. Heart failure can restrict lung volumes though pulmonary congestion, resulting in a reduced $D_{\text {LCO }}$. Pulmonary congestion usually increases capillary blood volume, possibly contributing to the higher $D_{\mathrm{LCO}} / V_{\mathrm{A}}$ values $[40,45]$. We showed that subjects with incident PRISm had a concomitant increase in BMI, independent of incident heart failure. Abdominal obesity may compromise lung function through mechanical effects of truncal obesity and/or metabolic effects of adipose tissue [44]. Type 2 diabetes showed a clear association with PRISm as well, but longitudinal studies were inconsistent regarding the direction of this association, and pathophysiological mechanisms remain unclear [46].

It is possible that subjects with PRISm represent an elderly population with normal age-related decline in $\mathrm{FEV}_{1}$ and FVC given the lower prevalence and higher effect estimates of mortality using GLI definitions (supplementary figure S3, supplementary table S4). Compared to GOLD definitions, GLI definitions reduce false-positive estimates of respiratory diseases in elderly subjects [47]. Small sample sizes in some subgroup analyses justified our decision to use GOLD definitions.

$\mathrm{FEV}_{1}$ and FVC decline rates were lower in subjects with PRISm-P1 than in others at P1, similar to previous findings $[18,22]$. One would rather expect an excess in lung function decline to accompany the higher mortality seen in PRISm. The measurement of lung function decline based on P1 and P2 has limitations. Subjects with PRISm with fast lung function decline were more likely to die prior to re-examination. This was in contrast to subjects with PRISm with a slower decline rate, who were more likely to participate in P2 of the study, resulting in a healthy survivor bias. This informative censoring bias of the most severe cases resulted in an underestimation of decline rates in subjects with prevalent PRISm 
(at P1). Subjects with incident PRISm between P1 and P2 or subjects with PRISm at P2 showed larger decline rates. Moreover, becasue loss of lung function is generally more accelerated in early stages of COPD, subjects with PRISm, characterised by even lower FEV 1 and FVC than COPD GOLD 1, have less potential to lose lung function [48]. Finally, heterogeneity within subjects with PRISm is large and some subjects even had improved $\mathrm{FEV}_{1}$ and FVC. Some of the changes between P1 and P2 in this group may have been the consequence of regression to the mean. Excessive lung function decline was seen in disadvantageous transitions (i.e. incident PRISm and incident COPD). Subjects with incident PRISm, showing the most severe decline rates, had the highest mortality rate, followed by subjects with persistent PRISm, despite their normal rates of lung function decline. Some subjects with persistent PRISm may be individuals who did not attain maximal lung function in early adulthood and experience typical age-related pulmonary decline. Lower total lung capacities on computed tomography images in PRISm $[18,21]$ and increased cardiopulmonary mortality in individuals with lower $\mathrm{FEV}_{1}$ and FVC values at young adulthood [49] confirm these findings. Absent emphysema $[18,21]$ and preserved $D_{\mathrm{LCO}} / V_{\mathrm{A}}$ in the current study support the hypothesis of at least some subjects with PRISm having smaller lung volumes but intact lung parenchyma.

Strengths of our study are its population-based setting, the long-term longitudinal follow-up, the large sample size and the availability of heart failure and diffusion capacity data. Limitations are the use of pre-bronchodilator spirometry. Actual restriction of pulmonary volumes, defined as a significant decrease in total lung capacity, is ideally diagnosed by additional pulmonary function tests encompassing body plethysmography or multi-breath dilution techniques using nitrogen or helium [50]. Further, through drop out and death of severely ill participants between the study visits, the longitudinal part of this study is susceptible to healthy survivor bias. Finally, despite the large sample size of the overall population, some subgroup analyses included very small numbers (e.g. cause-specific mortality such as cardiovascular deaths in PRISm $(n=15)$ and mortality rates in PRISm longitudinal subgroups such as persistent PRISm $(n=31))$.

To conclude, we provide evidence that PRISm is associated with impaired outcomes in terms of increased all-cause and cardiovascular mortality. Moreover, we demonstrated that PRISm consists of a heterogeneous population encompassing at least three subsets: a first subset who develops COPD during follow-up, a second subset with high cardiovascular burden and early mortality, and a third subset with persistent PRISm but normal lung function decline rates. These results emphasise the importance of recognising PRISm in clinical settings.

Acknowledgements: The authors thank the study participants, the staff from the Rotterdam Study and the participating general practitioners.

Conflict of interest: S.R.A. Wijnant reports grants from GalaxoSmithKline (award), outside the submitted work. E. de Roos has nothing to disclose. M. Kavousi has nothing to disclose. B.H. Stricker has nothing to disclose. N. Terzikhan has nothing to disclose. L. Lahousse reports grants from AstraZeneca and Chiesi (both awards), and expert consultation for Boehringer Ingelheim and Novartis, outside the submitted work. G.G. Brusselle reports personal fees from AstraZeneca (advisory boards and lecture fees), Boehringer Ingelheim (advisory boards and lecture fees), Chiesi (advisory boards and lecture fees), GlaxoSmithKline (advisory boards and lecture fees), Novartis (advisory boards and lecture fees), Sanofi (advisory boards) and Teva (advisory boards and lecture fees), outside the submitted work.

Support statement: This research was funded by the "Funds for Scientific Research Flanders (Fonds voor Wetenschappelijk Onderzoek Vlaanderen)", grant number 3G037618. The Rotterdam Study is supported by the Erasmus MC and Erasmus University Rotterdam; the Netherlands Organisation for Scientific Research (NWO) (grant no. 918-46-615); the Netherlands Organisation for Health Research and Development (ZonMW); the Research Institute for Diseases in the Elderly (RIDE); the Netherlands Genomics Initiative; the Ministry of Education, Culture and Science; the Ministry of Health, Welfare and Sports; the European Commission (DG XII; grant no. 601055); and the Municipality of Rotterdam. The funding sources had no involvement in the collection, analysis, writing, interpretation or the decision to submit the paper for publication. Funding information for this article has been deposited with the Crossref Funder Registry.

\section{References}

1 Kalhan R, Dransfield MT, Colangelo LA, et al. Respiratory symptoms in young adults and future lung disease. The CARDIA lung study. Am J Respir Crit Care Med 2018; 197: 1616-1624.

2 Guerra S, Sherrill DL, Venker C, et al. Morbidity and mortality associated with the restrictive spirometric pattern: a longitudinal study. Thorax 2010; 65: 499-504.

3 Sood A, Petersen H, Qualls C, et al. Spirometric variability in smokers: transitions in COPD diagnosis in a five-year longitudinal study. Respir Res 2016; 17: e147.

4 Kalhan R, Tran BT, Colangelo LA, et al. Systemic inflammation in young adults is associated with abnormal lung function in middle age. PLoS One 2010; 5: e11431.

5 Iyer VN, Schroeder DR, Parker KO, et al. The nonspecific pulmonary function test: longitudinal follow-up and outcomes. Chest 2011; 139: 878-886.

6 Vaz Fragoso CA, Gill TM, McAvay G, et al. Respiratory impairment and mortality in older persons: a novel spirometric approach. J Investig Med 2011; 59: 1089-1095. 
7 Jankowich $\mathrm{M}$, Elston B, Liu Q, et al. Restrictive spirometry pattern, cardiac structure and function, and incident heart failure in African Americans. The Jackson Heart Study. Ann Am Thorac Soc 2018; 15: 1186-1196.

8 Lutsey PL, Chen N, Mirabelli MC, et al. Impaired lung function, lung disease and risk of incident dementia. Am J Respir Crit Care Med 2018; 199: 1385-1396.

9 Kubota Y, Folsom AR, Matsushita K, et al. Prospective study of lung function and abdominal aortic aneurysm risk: the Atherosclerosis Risk in Communities study. Atherosclerosis 2018; 268: 225-230.

10 Sumida K, Kwak L, Grams ME, et al. Lung function and incident kidney disease: the Atherosclerosis Risk in Communities (ARIC) study. Am J Kidney Dis 2017; 70: 675-685.

11 Kubota Y, London SJ, Cushman M, et al. Lung function, respiratory symptoms and venous thromboembolism risk: the Atherosclerosis Risk in Communities Study. J Thromb Haemost 2016; 14: 2394-2401.

12 Ford ES, Mannino DM National Health and Nutrition Examination Survey Epidemiologic Follow-up Study Prospective association between lung function and the incidence of diabetes: findings from the National Health and Nutrition Examination Survey Epidemiologic Follow-up Study. Diabetes Care 2004; 27: 2966-2970.

13 Johnston AK, Mannino DM, Hagan GW, et al. Relationship between lung function impairment and incidence or recurrence of cardiovascular events in a middle-aged cohort. Thorax 2008; 63: 599-605.

14 Choudhary G, Jankowich M, Wu WC. Elevated pulmonary artery systolic pressure predicts heart failure admissions in African Americans: Jackson Heart Study. Circ Heart Fail 2014; 7: 558-564.

15 Lahousse L, Vernooij MW, Darweesh SKL, et al. Chronic obstructive pulmonary disease and cerebral microbleeds. The Rotterdam Study. Am J Respir Crit Care Med 2013; 188: 783-788.

16 Scarlata S, Pedone C, Fimognari FL, et al. Restrictive pulmonary dysfunction at spirometry and mortality in the elderly. Respir Med 2008; 102: 1349-1354.

17 Mannino DM, Holguin F, Pavlin BI, et al. Risk factors for prevalence of and mortality related to restriction on spirometry: findings from the First National Health and Nutrition Examination Survey and follow-up. Int $J$ Tuberc Lung Dis 2005; 9: 613-621.

18 Wan ES, Fortis S, Regan EA, et al. Longitudinal phenotypes and mortality in preserved ratio impaired spirometry in the COPDGene study. Am J Respir Crit Care Med 2018; 198: 1397-1405.

19 Honda Y, Watanabe T, Shibata Y, et al. Impact of restrictive lung disorder on cardiovascular mortality in a general population: the Yamagata (Takahata) study. Int J Cardiol 2017; 241: 395-400.

20 Park HJ, Byun MK, Rhee CK, et al. Significant predictors of medically diagnosed chronic obstructive pulmonary disease in patients with preserved ratio impaired spirometry: a 3-year cohort study. Respir Res 2018; 19: e185.

21 Diaz AA, Strand M, Coxson HO, et al. Disease severity dependence of the longitudinal association between CT lung density and lung function in smokers. Chest 2018; 153: 638-645.

22 Siddharthan T, Grigsby $\mathrm{M}$, Miele $\mathrm{CH}$, et al. Prevalence and risk factors of restrictive spirometry in a cohort of Peruvian adults. Int J Tuberc Lung Dis 2017; 21: 1062-1068.

23 Wijnant SRA, Lahousse L, De Buyzere ML, et al. Prevalence of asthma and COPD and blood eosinophil count in a middle-aged Belgian population. J Clin Med 2019; 8: e1122.

24 van den Borst B, Gosker HR, Zeegers MP, et al. Pulmonary function in diabetes: a metaanalysis. Chest 2010; 138: 393-406.

25 Vatrella A, Calabrese C, Mattiello A, et al. Abdominal adiposity is an early marker of pulmonary function impairment: findings from a Mediterranean Italian female cohort. Nutr Metab Cardiovasc Dis 2016; 26: 643-648.

26 Amaral AF, Coton S, Kato B, et al. Tuberculosis associates with both airflow obstruction and low lung function: BOLD results. Eur Respir J 2015; 46: 1104-1112.

27 Wannamethee SG, Shaper AG, Rumley A, et al. Lung function and risk of type 2 diabetes and fatal and nonfatal major coronary heart disease events: possible associations with inflammation. Diabetes Care 2010; 33: 1990-1996.

28 Thyagarajan B, Jacobs DR, Apostol GG, et al. Plasma fibrinogen and lung function: the CARDIA study. Int $J$ Epidemiol 2006; 35: 1001-1008.

29 Mannino DM, Ford ES, Redd SC. Obstructive and restrictive lung disease and markers of inflammation: data from the third national health and nutrition examination. Am J Med 2003; 114: 758-762.

30 Berger KI, Reibman J, Oppenheimer BW, et al. Lessons from the World Trade Center disaster: airway disease presenting as restrictive dysfunction. Chest 2013; 144: 249-257.

31 Mukherjee S, Roychoudhury S, Siddique S, et al. Respiratory symptoms, lung function decrement and chronic obstructive pulmonary disease in pre-menopausal Indian women exposed to biomass smoke. Inhal Toxicol 2014; 26: 866-872.

32 Ikram MA, Brusselle GGO, Murad SD, et al. The Rotterdam Study: 2018 update on objectives, design and main results. Eur J Epidemiol 2017; 32: 807-850.

33 Celli BR, MacNee W, Agusti A, et al. Standards for the diagnosis and treatment of patients with COPD: a summary of the ATS/ERS position paper. Eur Respir J 2004; 23: 932-946.

34 Graham BL, Brusasco V, Burgos F, et al. 2017 ERS/ATS standards for single-breath carbon monoxide uptake in the lung. Eur Respir J 2017; 49: e1600016.

35 Quanjer PH, Stanojevic S, Cole TJ, et al. Multi-ethnic reference values for spirometry for the 3-95-yr age range: the global lung function 2012 equations. Eur Respir J 2012; 40: 1324-1343.

36 Leening MJG, Kavousi M, Heeringa J, et al. Methods of data collection and definitions of cardiac outcomes in the Rotterdam Study. Eur I Epidemiol 2012; 27: 173-185.

37 de Roos EW, Lahousse L, Verhamme KMC, et al. Asthma and its comorbidities in middle-aged and older adults; the Rotterdam Study. Respir Med 2018; 139: 6-12.

38 Eurostat. Revision of the European Standard Population - Report of Eurostat's task force - 2013 edition. Luxembourg, European Union, 2013.

39 Olson TP, Beck KC, Johnson BD. Pulmonary function changes associated with cardiomegaly in chronic heart failure. J Card Fail 2007; 13: 100-107.

40 Magnussen $\mathrm{H}$, Canepa $\mathrm{M}$, Zambito $\mathrm{PE}$, et al. What can we learn from pulmonary function testing in heart failure? Eur J Heart Fail 2017; 19: 1222-1229.

41 Ball L, Costantino F, Pelosi P. Postoperative complications of patients undergoing cardiac surgery. Curr Opin Crit Care 2016; 22: 386-392. 
42 Wan ES, Castaldi PJ, Cho $\mathrm{MH}$, et al. Epidemiology, genetics, and subtyping of preserved ratio impaired spirometry (PRISm) in COPDGene. Respir Res 2014; 15: e89.

43 Ford ES, Cunningham TJ, Mercado CI. Lung function and metabolic syndrome: findings of National Health and Nutrition Examination Survey 2007-2010. J Diabetes 2014; 6: 603-613.

44 Leone N, Courbon D, Thomas F, et al. Lung function impairment and metabolic syndrome. Am J Respir Crit Care Med 2009; 179: 509-516.

45 Deis $\mathrm{T}$, Balling $\mathrm{L}$, Rossing $\mathrm{K}$, et al. Lung diffusion capacity in advanced heart failure: relation to central haemodynamics and outcome. ESC Heart Fail 2019; 6: 379-387.

46 Klein OL, Krishnan JA, Glick S, et al. Systematic review of the association between lung function and type 2 diabetes mellitus. Diabet Med 2010; 27: 977-987.

47 Stanojevic S, Quanjer P, Miller MR, et al. The Global Lung Function Initiative: dispelling some myths of lung function test interpretation. Breathe 2013; 9: 462-474.

48 Tantucci C, Modina D. Lung function decline in COPD. Int I Chron Obstruct Pulmon Dis 2012; 7: 95-99.

49 Vasquez MM, Zhou M, Hu C, et al. low lung function in young adult life is associated with early mortality. Am J Respir Crit Care Med 2017; 195: 1399-1401.

50 Tantucci C, Bottone D, Borghesi A, et al. Methods for measuring lung volumes: is there a better one? Respiration 2016; 91: 273-280. 